\title{
Is asymptomatic postoperative venous thromboembolism associated with long-term survival in patients undergoing lung resection for malignancy?
}

Gileh-Gol Akhtar-Danesh, MD, ${ }^{\mathrm{a}}$ Ronny Ben-Avi, MD, ${ }^{\mathrm{b}, \mathrm{c}}$ John Agzarian, MD, MPH, FRSCS, ${ }^{\mathrm{a}, \mathrm{d}}$ and Yaron Shargall, BSc, MD, FRCSC, FCCP, ${ }^{\text {a,d }}$ Hamilton, Ontario, Canada, and Tiberias and Safed, Israel

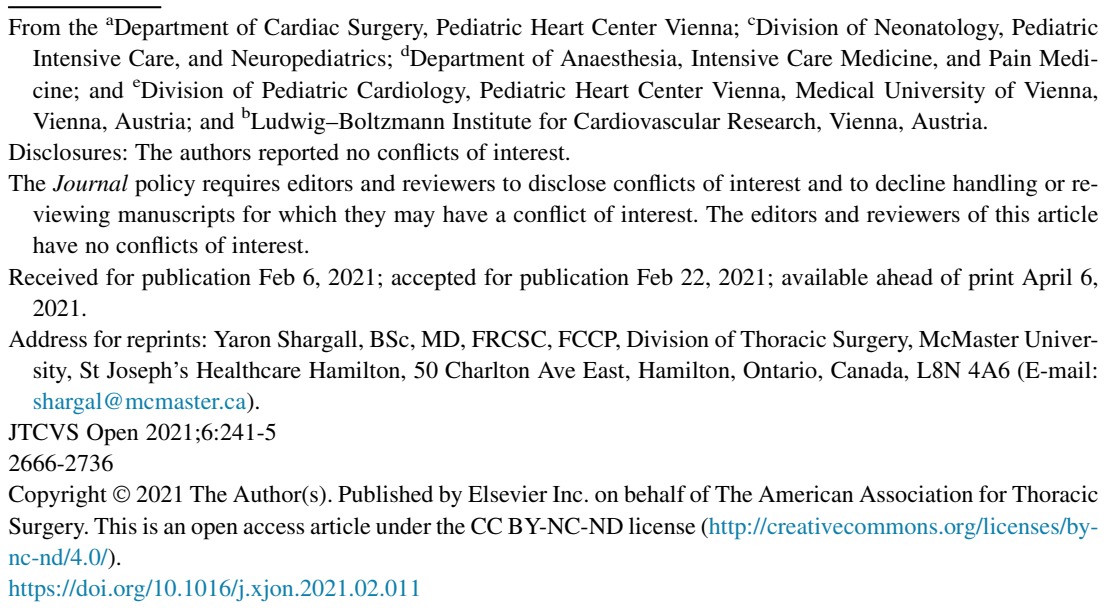

Venous thromboembolism (VTE), including deep-vein thrombosis (DVT) and pulmonary embolism (PE), is a significant cause of morbidity and mortality after lung resection. ${ }^{1}$ Previous studies have found postoperative VTEs are associated with increased 30 -day mortality. ${ }^{2}$ Although the majority of patients with lung cancer receive in-hospital prophylaxis, the American College of Surgeons National Surgical Quality Improvement Program reports that $44 \%$ of VTEs after lung resection occur after hospital discharge. ${ }^{2}$ Although general surgical oncology and orthopedic surgery have developed recommendations for extended, postdischarge prophylaxis, ${ }^{4}$ no such guidelines exist for lung cancer surgery. Furthermore, evidence suggests that VTE development after curative oncologic resections portends worse overall survival beyond the immediate postoperative period, potentially indicating a more aggressive malignancy.

Our group previously conducted a prospective cohort study across 2 tertiary hospitals in the Canadian province of Ontario and found a $12 \%$ incidence of screeningdetected postoperative VTEs, all diagnosed postdischarge. ${ }^{1}$ In light of evidence suggesting VTEs are associated with poor oncologic outcomes, we conducted a follow-up analysis to examine the relationship between postoperative VTEs and long-term survival.

\section{METHODS}

The original study recruited patients undergoing lung cancer resection across 2 tertiary centers in Ontario. ${ }^{1}$ Patients older than the age of 18 years undergoing lung resection were included. All patients received in-hospital pharmacologic and mechanical prophylaxis, including graduated compression

\section{RESULTS}

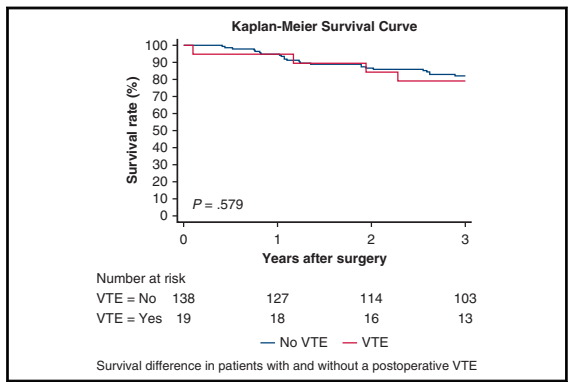

Survival difference in patients with and without a postoperative VTE.

CENTRAL MESSAGE

This study highlights that with regular venous thromboembolism (VTE) screening and subsequent treatment, postoperative thrombotic events may not impact the long-term survival of lung cancer patients.

See Commentaries on pages 246 and 248.

stockings and chemical prophylaxis with daily subcutaneous low-molecular weight heparin, or twice-daily unfractionated heparin. All study patients underwent screening computed tomography pulmonary angiography and bilateral above-knee lower-limb venous Doppler ultrasonography at 30 days postoperatively. Screening of asymptomatic patients was conducted only for study patients and is not standard of care. Patients with previous thrombotic events or on therapeutic anticoagulation were excluded.

For the present study, patients were examined with a median follow-up of 3.6 years after surgery. Patients with postoperative VTEs were compared with those without VTE. We used a proportional hazard Cox regression to compare survival between the groups. Age, sex, smoking status, and comorbidities were included in the univariate analysis. Variables that achieved significance were then included in the multivariable regression. Outcomes of interest were cancer recurrence and overall survival. Importantly, $22 \%$ of patients underwent pulmonary metastatectomy with a non-lung primary malignancy. Given the small number of total patients, all patients were included in the final survival curve. The patients provided informed consent for the publication of the study data.

The original analysis included 157 patients; 12\% $(n=19)$ developed a postoperative VTE. One death from 
TABLE 1. Baseline characteristics of the original cohort $(N=157)^{1}$

\begin{tabular}{|c|c|c|c|c|}
\hline & No postoperative VTE $(n=138)^{*}$ & Postoperative VTE $(\mathrm{n}=19)$ & Total $\dagger(\mathbf{N}=\mathbf{1 5 7})$ & $P$ value \\
\hline Age, y & $66.25 \pm 8.88$ & $69.05 \pm 11.51$ & $66.55 \pm 9.24$ & .216 \\
\hline Sex (male) & $62(44.92)$ & $10(52.63)$ & $72(45.86)$ & .626 \\
\hline$\%$ Predicted FEV 1 & $72.34(32.68)$ & $82.50(35.11)$ & $73.51(33.02)$ & .220 \\
\hline$\%$ Predicted DLCO & $72.07(19.88)$ & $67.0(14.13)$ & $71.48(19.33)$ & .326 \\
\hline Charlson Comorbidity Index & $2.19 \pm 2.07$ & $2.42 \pm 2.24$ & $2.22 \pm 2.08$ & .649 \\
\hline Length of stay, d & $6(3-24)$ & $5(1-5)$ & $5(1-24)$ & .185 \\
\hline \multicolumn{5}{|l|}{ Caprini score } \\
\hline $3-4$ & $27(19.56)$ & $2(10.52)$ & $29(18.47)$ & .441 \\
\hline $5+$ & $111(80.43)$ & $17(89.47)$ & $128(81.52)$ & \\
\hline \multicolumn{5}{|l|}{ Smoking status } \\
\hline Never smoker & $26(83.9)$ & $5(16.1)$ & $31(19.7)$ & .441 \\
\hline Former smoker & $79(90.8)$ & $8(9.2)$ & $87(55.4)$ & \\
\hline Current smoker & $32(84.2)$ & $6(15.8)$ & $38(24.2)$ & \\
\hline \multicolumn{5}{|l|}{ Tumor pathology } \\
\hline T1a & $27(81.8)$ & $6(18.2)$ & $33(26.0)$ & \\
\hline $\mathrm{T} 1 \mathrm{~b}$ & $18(85.7)$ & $3(14.3)$ & $21(16.5)$ & \\
\hline $\mathrm{T} 2 \mathrm{a}$ & $35(83.3)$ & $7(16.7)$ & $42(33.1)$ & .513 \\
\hline $\mathrm{T} 2 \mathrm{~b}$ & $11(91.7)$ & $1(8.3)$ & $12(9.4)$ & \\
\hline $\mathrm{T} 3$ & $16(100)$ & 0.0 & $16(12.6)$ & \\
\hline $\mathrm{T} 4$ & $3(100)$ & 0.0 & $3(2.4)$ & \\
\hline \multicolumn{5}{|l|}{ Lymph node pathology } \\
\hline $\mathrm{NX}$ & $4(100)$ & 0.0 & $4(3.1)$ & \\
\hline No & $79(85.9)$ & $13(14.1)$ & $92(72.4)$ & .566 \\
\hline N1 & $21(91.3)$ & $2(8.7)$ & $23(18.1)$ & \\
\hline $\mathrm{N} 2$ & $6(0.8)$ & $2(0.2)$ & $8(6.3)$ & \\
\hline \multicolumn{5}{|l|}{ Pathologic stage (TMN) } \\
\hline IA & $37(26.81)$ & $7(36.84)$ & $44(28.03)$ & \\
\hline IB & $31(22.46)$ & $6(31.58)$ & 37 (23.57) & \\
\hline IIA & $14(10.14)$ & $1(5.26)$ & $15(9.55)$ & $-\ddagger$ \\
\hline IIB & $7(5.07)$ & $1(5.26)$ & $8(5.10)$ & \\
\hline IIIA & $16(11.59)$ & $2(10.53)$ & $18(11.46)$ & \\
\hline IIIB & $4(2.90)$ & $0(0)$ & $4(2.55)$ & \\
\hline Lung metastases & $21(15.22)$ & $2(10.53)$ & $23(14.65)$ & \\
\hline \multicolumn{5}{|l|}{ Histology } \\
\hline Squamous cell & $29(21.01)$ & $4(21.05)$ & $33(21.01)$ & .827 \\
\hline Adenocarcinoma & $63(45.65)$ & $10(52.63)$ & $73(46.50)$ & \\
\hline Other & $45(32.61)$ & $5(26.32)$ & $50(31.85)$ & \\
\hline \multicolumn{5}{|l|}{ Resection } \\
\hline Pneumonectomy & $6(4.35)$ & $0(0)$ & $6(3.82)$ & \\
\hline Bilobectomy & $2(1.45)$ & $0(0)$ & $2(1.27)$ & $-\ddagger$ \\
\hline Lobectomy & 87 (63.04) & 15 (78.96) & $102(64.97)$ & \\
\hline Sublobar & 43 (31.16) & $4(21.05)$ & 47 (29.93) & \\
\hline \multicolumn{5}{|l|}{ Surgical approach } \\
\hline VATS & $76(55.07)$ & 9 (47.37) & 85 (54.14) & \\
\hline Thoracotomy & $56(40.58)$ & $10(52.63)$ & $66(42.04)$ & .452 \\
\hline Robotic & $6(4.35)$ & $0(0)$ & $6(3.82)$ & \\
\hline
\end{tabular}

Groups were compared using $t$ tests and $\chi^{2}$ tests as appropriate. VTE, Venous thromboembolism; $F E V_{l}$, forced expiratory volume in $1 \mathrm{~s} ; D L C O$, diffusion capacity of the lungs for carbon monoxide; VATS, video-assisted thoracoscopic surgery. *Values represent $\mathrm{n}(\%)$, mean \pm standard deviation, or median (range) unless otherwise specified. $\dagger$ Total for all variables may not add up to 157 due to missing data. Ł๋Due to small sample size, $P$ value is not reliable. 
TABLE 2. Survival rate (\%) over time for patients with and without a postoperative screen-detected VTE

\begin{tabular}{lccc}
\hline Time (years since surgery) & Number at risk & Survival rate $(\%)$ & 95\% confidence interval \\
\hline No VTE & & & NA \\
0 & 138 & 100 & $89.5-97.5$ \\
1 & 127 & 94.9 & $79.6-91.4$ \\
2 & 114 & 86.7 & $74.4-87.6$ \\
3 & 103 & 82.0 & $67.2-83.1$ \\
4 & 12 & 76.3 & NA \\
VTE & & & $68.1-99.2$ \\
0 & 19 & 100 & $58.7-94.6$ \\
1 & 18 & 94.7 & $53.2-91.5$ \\
2 & 16 & 84.2 & $17.6-80.2$ \\
3 & 13 & 80.0 & 53.4 \\
4
\end{tabular}

VTE, Venous thromboembolism; $N A$, not available.

massive PE resulted in a 5\% 30-day mortality rate from VTE in the VTE group, whereas none of the non-VTE group died. ${ }^{1}$ Only 4 patients $(21.1 \%)$ were symptomatic. ${ }^{1}$
Univariate analysis showed no difference between patients with and without a VTE with regards to baseline characteristics (Table 1).

TABLE 3. Proportional hazard Cox regression analysis of survival for all patients (VTE + no VTE)

\begin{tabular}{|c|c|c|c|c|c|}
\hline & $\mathbf{n}$ & Univariable HR $(95 \%$ CI $)$ & $P$ value & Multivariable HR $(95 \%$ CI $)$ & $P$ value \\
\hline Age, $y$ & 157 & $1.02(0.98-1.06)$ & .239 & - & - \\
\hline Sex & & & .720 & - & - \\
\hline Female & 72 & Reference & & & \\
\hline Male & 85 & $1.12(0.60-2.08)$ & & & \\
\hline Smoking history & & & .832 & - & - \\
\hline No & 127 & Reference & & & \\
\hline Yes & 30 & $1.09(0.50-2.36)$ & & & \\
\hline Any VTE & & & .501 & - & - \\
\hline No & 138 & Reference & & & \\
\hline Yes & 19 & $1.34(0.56-3.21)$ & & & \\
\hline Pathologic stage & 149 & $1.18(1.05-1.32)$ & .004 & $1.17(1.05-1.31)$ & .005 \\
\hline Histology & & & .336 & - & - \\
\hline Squamous cell & 33 & Reference & - & & \\
\hline Adenocarcinoma & 73 & $0.92(0.40-2.16)$ & .858 & & \\
\hline Carcinoid & 12 & 0.00 & .974 & & \\
\hline Metastatic & 23 & $1.87(0.74-4.76)$ & .184 & & \\
\hline Mixed & 15 & $1.90(0.66-5.47)$ & .235 & & \\
\hline Surgery & & & .742 & - & - \\
\hline Pneumonectomy & 6 & Reference & - & & \\
\hline Lobectomy & 104 & $0.60(0.14-2.56)$ & .495 & & \\
\hline Segmentectomy & 27 & $0.82(0.17-3.80)$ & .798 & & \\
\hline Wedge & 20 & $0.50(0.10-2.73)$ & .424 & & \\
\hline $\mathrm{FEV}_{1}$ & 149 & $0.99(0.98-1.01)$ & .362 & - & - \\
\hline DLCO & 146 & $0.98(0.96-1.00)$ & .125 & - & - \\
\hline VATS & & & .036 & NS & NS \\
\hline No & 71 & Reference & & & \\
\hline Yes & 84 & $0.51(0.27-0.95)$ & & & \\
\hline
\end{tabular}


TABLE 3. Continued

\begin{tabular}{|c|c|c|c|c|c|}
\hline & $\mathbf{n}$ & Univariable HR $(95 \%$ CI $)$ & $P$ value & Multivariable HR $(95 \%$ CI $)$ & $P$ value \\
\hline CVA & & & .446 & - & - \\
\hline No & 152 & Reference & & & \\
\hline Yes & 5 & $1.74(0.42-7.21)$ & & & \\
\hline PVD & & & .076 & NS & NS \\
\hline No & 148 & Reference & & & \\
\hline Yes & 9 & $2.34(0.91-5.98)$ & & & \\
\hline CAD & & & .951 & - & - \\
\hline No & 137 & Reference & & & \\
\hline Yes & 20 & $0.97(0.38-2.48)$ & & & \\
\hline Diabetes & & & .066 & NS & NS \\
\hline No & 134 & Reference & & & \\
\hline Yes & 23 & $0.26(0.06-1.09)$ & & & \\
\hline Obesity & & & .724 & - & - \\
\hline No & 137 & Reference & & & \\
\hline Yes & 20 & $1.17(0.49-2.80)$ & & & \\
\hline CKD & & & .484 & - & - \\
\hline No & 134 & Reference & & & \\
\hline Yes & 22 & $1.34(0.60-3.02)$ & & & \\
\hline
\end{tabular}

$H R$, Hazard ratio; $C I$, confidence interval; VTE, venous thromboembolism; $F E V_{l}$, forced expiratory volume in $1 \mathrm{~s} ; D L C O$, diffusion capacity of the lungs for carbon monoxide; $V A T S$, video-assisted thoracoscopic surgery; $C V A$, cerebrovascular accident; $P V D$, peripheral vascular disease; $C A D$, coronary artery disease; $N S$, not significant; $C K D$, chronic kidney disease.

Long-term follow-up was complete for all patients and showed no difference in cancer recurrence between patients with and without a VTE $35 \%$ and $32 \%$, respectively, $P=1.000$; median follow-up 3.6 years). Results were unchanged when DVT and PE were analyzed separately. There was no difference in overall or disease-specific survival between the 2 groups (Tables 2 and 3, Figure 1). This effect persisted after stratification by disease stage and patient characteristics.

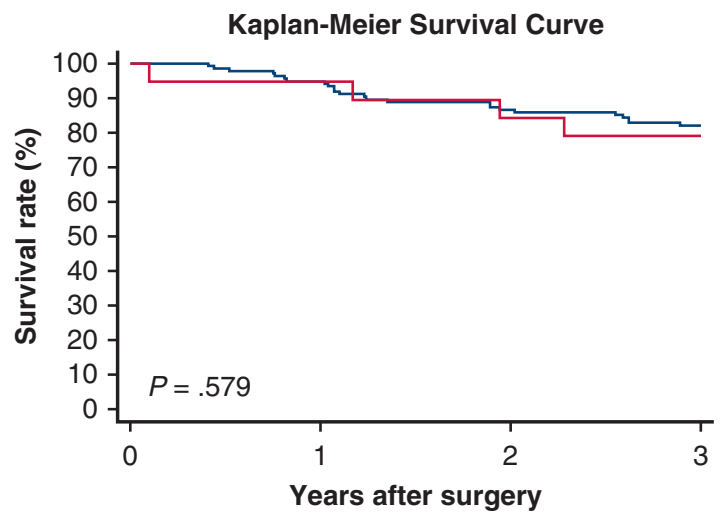

Number at risk

VTE $=$ No 138

$\mathrm{VTE}=$ Yes 19

$\begin{array}{ccc}127 & 114 & 103 \\ 18 & 16 & 13 \\ - \text { No VTE } & - \text { VTE } & \end{array}$

FIGURE 1. Overall long-term survival in patients undergoing lung cancer surgery with and without a postoperative screening-detected VTE. *Confidence intervals reported in Table 2. VTE, Venous thromboembolism.

\section{DISCUSSION}

This study found no difference in the long-term survival of patients with lung cancer based on postoperative VTE development. These results stand in contrast to previous evidence suggesting worse overall survival in patients with a postoperative VTE. ${ }^{5}$ Notably, this study captured asymptomatic, screening-detected VTEs, prompting treatment of patients who may have not manifested clinical evidence of DVT/PE and remained untreated. It is possible that our findings are due to early identification and subsequent treatment of patients with subclinical VTEs, preventing longterm morbidity from undetected DVT/PEs.

The strengths of this study include long-term and granular follow-up of patients post-lung resection. The small sample size is the major limitation, as it increases the likelihood of type II errors. Furthermore, the inclusion of pulmonary metastases in the survival curve decreases the generalizability of results to patients with lung cancer. Finally, bleeding complications after the initiation of therapeutic anticoagulation in the VTE group were not tracked.

In conclusion, the present study found that with regular VTE screening and treatment when an event is detected, postoperative VTEs may not impact the long-term survival of patients undergoing lung resection for malignancy. Rather, the morbidity and mortality of postoperative VTEs seems to lie in the short-term postoperative period. To reduce the impact of VTEs on long-term survival, screening for high-risk patients may be warranted to promote early diagnosis and treatment, as treated events are unlikely to impact long-term outcomes. Similar to surgical 
oncology, thoracic surgeons may consider extended postdischarge VTE prophylaxis for selected patient populations to prevent the development of thrombotic complications.

\section{References}

1. Agzarian J, Hanna WC, Schneider L, Schieman C, Finley CJ, Peysakhovich Y, et al. Postdischarge venous thromboembolic complications following pulmonary oncologic resection: an underdetected problem. J Thorac Cardiovasc Surg. 2016;151:992-9.
2. Thomas DC, Arnold BN, Hoag JR, Salazar MC, Detterbeck FC, Boffa DJ, et al Timing and risk factors associated with venous thromboembolism after lung cancer resection. Ann Thorac Surg. 2018;105:1469-75.

3. Kearon C, Akl EA, Ornelas J, Blaivas A, Jimenez D, Bounameaux H, et al. Antithrombotic therapy for VTE disease. Chest. 2016;149:315-52.

4. Lyman GH, Bohlke K, Khorana AA, Kuderer NM, Lee AY, Arcelus JI, et al. Venous thromboembolism prophylaxis and treatment in patients with cancer: American Society of Clinical Oncology clinical practice guideline update 2014 J Clin Oncol. 2015;33:654-6.

5. Auer RAC, Scheer AS, McSparron JI, Schulman AR, Tuorto S, Doucette S, et al Postoperative venous thromboembolism predicts survival in cancer patients. Ann Surg. 2012;255:963-70. 